\title{
Special issue on Additive manufacturing: progress in modeling and simulation with experimental validations in additive manufacturing
}

\author{
Wing Kam Liu ${ }^{1}$ Y Yung C. Shin ${ }^{2}$
}

Published online: 26 February 2018

(c) Springer-Verlag GmbH Germany, part of Springer Nature 2018

\section{Preface}

Additive manufacturing (AM) methods have great potential for promoting transformative manufacturing and product design in many fields across the vast spectrum of engineering and materials science. AM is one of the leading forms of advanced manufacturing, which enables direct computeraided design (CAD) to part production without part-specific tooling. Conventional applications, such as tooling, low production parts, biomedical devices and implants, aerospace components and rapid prototyping, all benefit from the flexibility that AM provides. The technology likewise can enable materials design and development of metamaterials and functional gradient materials, among many others. However a number of obstacles must be overcome before AM can be an industrially viable, widely accepted manufacturing technology. These obstacles include an understanding of the relationship between the process parameters and the resultant properties of the parts built by AM, the robustness of the process, and in-process monitoring and control and its associated costs. The goal of this special issue is devoted to capturing the theoretical and computational approaches to additive manufacturing as well as some critical experimental work going on in the leading groups of researchers to overcome these obstacles.

The first paper entitled Deriving process-structureproperty relationships for additive manufacturing with datadriven multiscale multi-physics material models summa-

Wing Kam Liu

w-liu@northwestern.edu

1 Walter P. Murphy Professor of Mechanical and Civil Engineering, Department of Mechanical Engineering, Northwestern University, 2145 Sheridan Rd., Evanston, IL 60208-3111, USA

2 Donald A. \& Nancy G. Roach Professor of Advanced Manufacturing, School of Mechanical Engineering, Purdue University, 585 Purdue Mall, West Lafayette, IN 47907, USA rizes recent work on multi-scale multi-physics modeling of process-structure-property relationships for AM. The multi-scale process modeling consists of: an electron beam heat source model derived from micro-scale simulations of electron-atom interactions, powder-scale powder spreading and thermal-fluid flow models to simulate manufacturing processes of multiple powder layers, and an efficient partscale Finite Element heat transfer model. The predicted temperature fields are used in a Cellular Automaton model to simulate grain growth during solidification. Microstructural information is used to predict the mechanical behaviorstress-strain response and fatigue crack incubation potency are given as case studies-using a crystal plasticity constitutive law implemented in a new data-driven reduced order method called Self-consistent Clustering Analysis. Preliminary experimental results show promising similarity to simulation results. Finally, further use of data-mining techniques is proposed to close the cycle of design-predictoptimize.

The second paper entitled A Parallelized 3D CAFE Model for Grain Growth during Additive Manufacturing develops and applies a parallelized cellular automaton/finite element (CAFE) implementation for the prediction of grain structure resulting from the complex thermal field in the additive manufacturing process. The parallel implementation decomposes the geometry spatially, allowing distribution of both memory and computational load and allowing large-scale 3D cell grids. The approach is shown to scale well in parallel, although the fundamentally localized solidification process places limits on scalability due to load balance issues. The authors demonstrate the effect of refining cell and time step sizes on the resulting microstructures. The method is applied to a selective electron beam melting process and predicts a complex 3D columnar microstructure that compares well with experimental characterization of additively manufactured material.

The third article entitled Simulation and Experimental Comparison of the Thermo-Mechanical History and $3 D$ 
Microstructure Evolution of 304L Stainless Steel Tubes Manufactured Using LENS presents computational methods to model the LENS process-which is a laser-based directed energy deposition technique. Finite element analysis is first used to simulate the thermal history, including the sharp thermal gradients caused by the localized laser heat source. The thermal results are then coupled to a solid mechanics simulation that utilizes a temperature-dependent elasto-viscoplastic internal state variable model to predict the residual stresses produced during the process. The thermal history is also implemented in a Potts Kinetic Monte Carlo model to capture three-dimensional grain nucleation and evolution as the part is built. The resulting microstructure demonstrates an equiaxed-to-columnar transition, which is also present in experimental electron backscatter diffraction measurements.

The fourth paper Characterization of Metal Additive Manufacturing Surfaces using Synchrotron X-ray CT and Micromechanical Modeling presents the instantiation of an image-based micromechanical model that allows for the simulation of stress concentrations due to geometric inhomogeneity inherent to rough surfaces. In this work 3D images from Synchrotron-based X-ray microtomography ( $\mu$ XCT) measurements of Additive Manufactured (AM) parts have allowed for the analysis of the surface features that create hotspots, and a comparison between different rough surfaces. Two AM builds were completed with varying powder size distributions, and sections of the rough surfaces were analyzed with $\mu \mathrm{XCT}$. Simulations of uniaxial tension reveal stress hotspots are not due to the powder directly, and apparent differences in roughness do not necessarily affect the stress concentrations.

The fifth paper Experimental Validation of $3 D$ Printed Material Behaviors and their Influence on the Structural Topology Design verifies the anisotropic mechanical behaviors of 3D printed resin material and metal material by means of experimental tests. On this basis, this work studies the performance of topology optimized structures and metal lattice structures, which have been popularly presented in many successful applications. The experiments show anisotropic material properties and diversities of structural performance caused by printing direction. It is also proved that the lattice structure has the advantage of being insensitive to local defects and damage within a certain load range. However, this structural form also presents large uncertainties in structural strength due to the quality of the formed surface, internal defects and the like. The above evidence clearly shows that the behavior and instabilities of the material affect the structural design and optimization significantly. Consideration of this behavior and the matching design of the material and structural properties is therefore indispensable in improving the structural performance and practical significance of 3D printing.

The sixth paper entitled Prediction of Microstructure, Residual Stress, and Deformation in Laser Powder Bed Fusion Process presents modeling methods which have potential to be used to simulate the laser powder bed fusion (L-PBF) process for building large-scale parts. Modeling methods and tools, which can be used in a part level, are essential to allow engineers to fine tune the shape design and process parameters for L-PBF. A transient sequentially coupled thermal and metallurgical analysis method is developed to predict microstructure and hardness on L-PBF built high-strength, low-alloy steel parts. A sequentially coupled thermal and mechanical analysis method is developed to predict residual stress and deformation for an L-PBF build part. It was found that a line-heating model is not suitable for analyzing a large L-PBF built part. The layer heating method is a potential method for analyzing a large L-PBF built part. Experiments were conducted to validate the model predictions.

The seventh paper entitled Predictive Modeling Capabilities from Incident Powder and Laser to Mechanical Properties for Laser Directed Energy Deposition describes a comprehensive and integrated modeling framework, which can be used for predicting mechanical properties of additive manufactured parts by directed energy deposition processes as well as other additive manufacturing processes. The authors develop numerical models for powder flow and molten pool dynamics using CFD methods, the resultant microstructure during solidification using a novel hybrid cellular automata-phase field (CAPF) scheme, solid state phase transformation during repeated heating and cooling cycles using the solid-state kinetics model and finally hardness and residual stresses based on the thermal histories and resultant phase and microstructure. They also illustrate how different modules are connected and demonstrated successful case study results. The potential of the developed models as useful tools for designing the metal additive manufacturing processes to achieve the desired microstructure and mechanical properties is demonstrated.

\section{Guest Editors}

Wing Kam Liu, Northwestern University

Yung C. Shin, Purdue 